\title{
Influences of non-singular stresses on plane-stress near-tip fields for pressure-sensitive materials and applications to transformation toughened ceramics
}

\author{
Z.E.A. BEN-AOUN ${ }^{1}$ and J. PAN ${ }^{2}$ \\ 'Centre for Surface Transportation Technology, National Research Council, U89, Alert Road, Uplands, Ottawa, \\ Ontario KIA OR6, Canada \\ ${ }^{2}$ Department of Mechanical Engineering and Applied Mechanics, The University of Michigan, Ann Arbor, \\ MI 48109-2I25, USA
}

Received 20 January 1995; accepted in revised form 31 January 1996

\begin{abstract}
In this paper, we investigate the effects of the non-singular stress ( $T$ stress) on the mode I near-tip fields for elastic perfectly plastic pressure-sensitive materials under plane-stress and small-scale yielding conditions. The $T$ stress is the normal stress parallel to the crack faces. The yield criterion for pressure-sensitive materials is described by a linear combination of the effective stress and the hydrostatic stress. Plastic dilatancy is introduced by the normality flow rule. The results of our finite element computations based on a two-parameter boundary layer formulation show that the total angular span of the plastic sectors of the near-tip fields increases with increasing $T$ stress for materials with moderately large pressure sensitivity. The $T$ stress also has significant effects on the sizes and shapes of the plastic zones. The height of the plastic zone increases substantially as the $T$ stress increases, especially for materials with large pressure sensitivity. When the plastic strains are considered to be finite as for transformation toughened ceramics, the results of our finite element computations indicate that the phase transformation zones for strong transformation ceramics with large pressure sensitivity can be approximated by those for elastic-plastic materials with no limit on plastic strains. When the $T$ stress and the stress intensity factor IS are prescribed in the two-parameter boundary layer formulation to simulate the crack-tip constraint condition for a single-edge notch bend specimen of zirconia ceramics, our finite element computation shows a spear shape of the phase transformation zone which agrees well with the corresponding experimental observation.
\end{abstract}

\section{Introduction}

In classical Linear Elastic Fracture Mechanics (LEFM), it is assumed that the fracture processes occurring close to a crack tip are governed by the far-field stress intensity factor $K$. Then the elastic-plastic problem under small-scale yielding is replaced by a boundary layer formulation where the boundary condition is set to either the traction or displacement given by the elastic asymptotic crack-tip field which is scaled by the stress intensity factor $K$.

Larsson and Carlsson [1] investigated the plane-strain crack-tip fields for compact tension, bend, double edge-cracked, and center-cracked specimens by finite element computations. They found differences of 10 to 30 percent in fracture surface displacement, normal stress, and plastic zone size between the finite element solutions of the specimens. As suggested by Rice [2], Larsson and Carlsson [1] were able to eliminate the differences in the fields by introducing the non-singular stress term in their two-parameter boundary layer formulation where the remote traction or displacement is expressed in terms of $K$ and the transverse $T$ stress.

The $J$ integral (Rice [3]) and the HRR crack-tip fields (Hutchinson [4, 5]; Rice and Rosengren [6]) provide the basis for nonlinear fracture mechanics. An excellent review of the $J$-based fracture mechanics can be found in Hutchinson [7]. McMeeking and Parks [8] and 
Shih and German [9] investigated the requirement of one parameter characterization and the $J$-dominance of the crack-tip fields. Recently, Betegón and Hancock [10] attempted to characterize the crack-tip fields for hardening materials under mode I plane-strain conditions using $J$ and $T$. Their results show that geometries which maintain $J$-dominance are characterized by zero or positive $T$ stresses, while geometries with negative $T$ stresses can be characterized by $J$ and $T$ under fully yielded conditions. Al-Ani and Hancock [11] showed that edge-cracked bars lose $J$-dominance for the crack length to width ratios less than 0.3 in bending and 0.5 in tension. The loss of $J$-dominance can be explained by negative $T$ stresses, while geometries with positive $T$ stresses retain $J$-dominance under fully yielded conditions. The $T$ stress has been subsequently validated by experimental results as an appropriate parameter to characterize crack-tip constraint conditions even under fully yielded conditions (Hancock, Reuter, and Parks [12]).

Also the angular variations of the mode I near-tip stresses as functions of the $T$ stress were studied by Du and Hancock [13] for elastic perfectly plastic materials under plane strain conditions. Their results show that the crack-tip field appears to be an incomplete Prandtl field with elastic sectors bordering the crack faces. However, they did not attempt to characterize the angular variations of the near-tip stresses in their elastic sectors as in Dong and Pan [14] and Kim and Pan [15]. Kim and Pan [15] resolved the asymptotic structures of the mode I near-tip fields for pressure-sensitive materials under plane strain conditions. Their numerical results show that the solution of the elastic sector of finite stress given by Dong and Pan [14] are essential to obtain the complete angular variation of the near-tip stresses under small-scale yielding conditions. The numerical results of Kim and Pan [15] also show the effects of the $T$ stress on the near-tip fields and the sizes and shapes of plastic zones for pressure-sensitive materials.

O'Dowd and Shih [16, 17] investigated the $J-Q$ annulus concept using a two-parameter boundary layer formulation under plane strain conditions. Their work shows that $J$ sets the size scale over which large stresses and strains develop and $Q$ is the measurement of the stress triaxiality ahead of the tip. Negative (positive) $Q$ values mean that the hydrostatic stress is decreased (increased) by $Q \sigma_{0}$ from the $Q=0$ reference state (here, $\sigma_{0}$ being a reference stress). Therefore $Q$ provides a quantitative measure of the crack-tip constraint. The $J-Q$ characterization of the near-tip fields can be used from small-scale yielding to fully yielded conditions.

Most of the research discussed above on the constraint conditions for pressure-insensitive Mises materials and on the near-tip fields for pressure-sensitive materials are under plane strain conditions. There are asymptotic analyses of near-tip fields under plane stress conditions. For example, Hutchinson [5] obtained the asymptotic crack-tip field for power-law hardening and perfectly plastic Mises materials. Pan and Shih [18] and Pan [19] obtained the asymptotic crack-tip fields for orthotropic materials. Li and Pan [20] obtained the asymptotic crack-tip fields for pressure-sensitive materials. Recently, Ben Aoun and Pan [21] obtained the neartip fields for elastic perfectly plastic pressure-sensitive materials under small-scale yielding conditions by both finite element computations and asymptotic analyses.

In this paper, we study the effects of the non-singular stress on mode I near-tip fields for pressure-sensitive materials under plane-stress and small-scale yielding conditions. We first investigate the near-tip fields for elastic perfectly plastic pressure-sensitive materials by finite element methods using the two-parameter boundary layer formulation characterized by $K$ and $T$ under monotonically increasing proportional loading conditions. The asymptotic near-tip stress fields are assembled and compared with the finite element results. Then we 


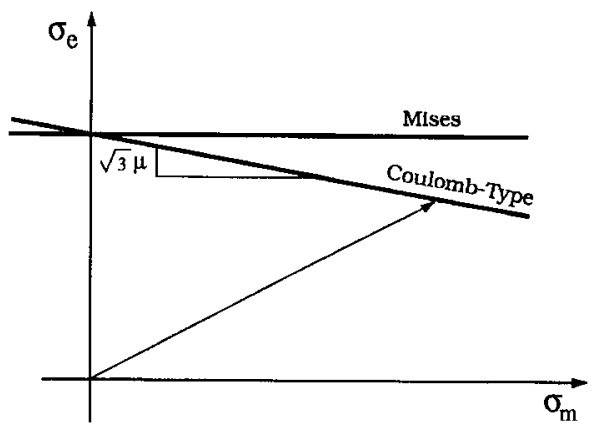

Figure 1. The Coulomb-type and Mises yield conditions.

consider the cases where the plastic strains are finite as the phase transformation strains in transformation toughened ceramics. We explore the near-tip stress and strain distributions for strong phase transformation ceramics where the phase transformation strains are much larger than the strains at the inception of phase transformation. We then compare the sizes and shapes of these phase transformation (plastic) zones with those of perfectly plastic materials. Finally, we investigate the phase transformation zone near a crack by the two-parameter boundary layer formulation characterized by $K$ and $T$ which represent the constraint conditions for a crack in a single-edge notch bend specimen of ceria-partially-stabilized zirconia polycrystals [22]. Our computational result is compared with the corresponding experimental result. It should be noted that the phase transformation zone near the crack in the zirconia ceramics appears to be a shape of a long narrow spear which has not been simulated by any theoretical or computational analysis.

\section{Constitutive laws}

In this study, we account for pressure-sensitive yielding or phase transformation by a Coulombtype criterion [23] which is a linear combination of the mean stress $\sigma_{m}\left(=\sigma_{k k} / 3\right)$ and the tensile effective stress $\sigma_{e}\left(=\left(3 s_{i j} s_{i j} / 2\right)^{1 / 2}\right.$ where $\left.s_{i j}=\sigma_{i j}-\sigma_{m} \delta_{i j}\right)$, as in [15]. Here, $\delta_{i j}$ is the Kronecker delta and subscripts $i, j$, and $k$ have the range of 1 to 3 . The Coulomb-type yield criterion is expressed as

$$
f\left(\sigma_{i j}\right)=\sigma_{e}+\sqrt{3} \mu \sigma_{m}=\sigma_{g e}=\sigma_{0}
$$

where $f\left(\sigma_{i j}\right)$ represents the yield surface in the stress space, $\sigma_{g e}$ is the generalized tensile effective stress, and $\mu$ represents the pressure sensitivity of the material. For perfectly plastic materials, $\sigma_{g e}$ is taken as a constant and is denoted as $\sigma_{0}$. The Coulomb-type yield condition and the familiar Mises yield condition are plotted in Figure 1. Note that the Coulomb-type yield criterion reduces to the Mises yield criterion when $\mu$ becomes zero. For HY-80, maraging, 4310 , and 4330 steels, Spitzig et al. [24, 25] showed that the values of $\mu$ lie between 0.014 and 0.064 . For polymers, Kinloch and Young [26] reported that the values of $\mu$ lie between 0.10 and 0.25 . For zirconia-containing ceramics, Chen [27] indicated that the values of $\mu$ for phase transformation are 0.55 and 0.77 for Mg-PSZ and Ce-TZP, respectively. Yu and Shetty [22] reported that the value of $\mu$ for phase transformation can go up to 0.93 for Ce-TZP. 


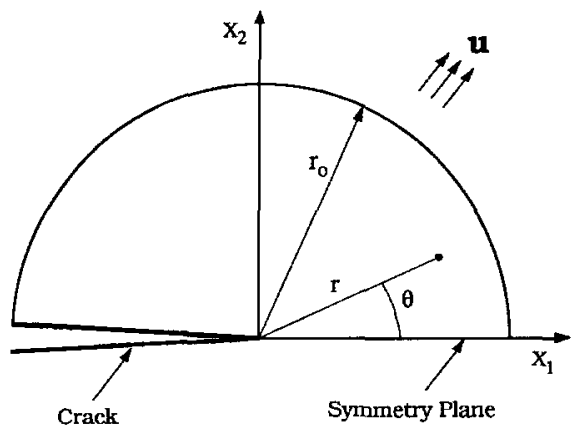

Figure 2. The coordinate conventions and finite element model.

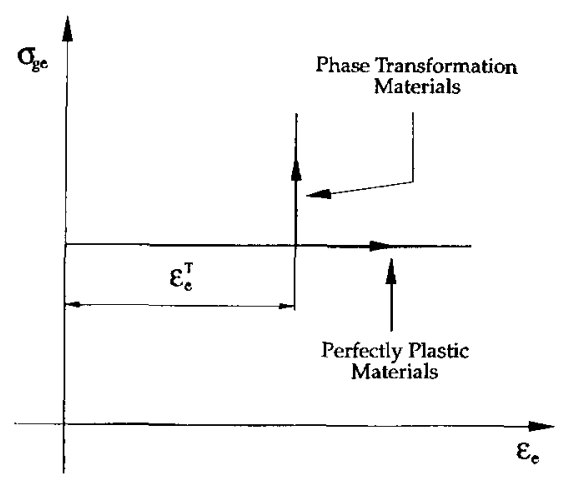

Figure 3. The generalized effective stress as a function of the effective plastic or phase transformation strain for perfectly plastic or phase transformation materials.

Here, we account for both the shear and hydrostatic stresses in the yield or phase transformation criterion. It should be noted that both shear and volumetric strains occur in the transformation from the tetragonal to monoclinic phase. Our phase transformation criterion differs from that in the previous studies of transformation toughening by McMeeking and Evans [28], Budiansky et al. [29], Rose [30], Amazigo and Budiansky [31], Stump and Budiansky [32], and Hom and McMeeking [33] where only the volumetric part of the phase transformation was considered. Lambropoulos [34] derived a constitutive formulation of plasticity type including the effects of shear for phase transformation ceramics and his results indicated that both shear and dilatational components of phase transformation contribute toughening based on the unperturbed linear elastic asymptotic crack-tip solution. Giannakopoulos and Olsson [35] recently investigated the influence of the nonsingular stress term on transformation toughening based on the pure dilatational phase transformation criterion and maximum principal stress phase transformation criterion.

We consider a planar crack problem as shown in Figure 2 where the Cartesian coordinates $x_{1}$ and $x_{2}$ represent the in-plane coordinates, the polar coordinates $r$ and $\theta$ are centered at the crack tip, and $x_{3}$ represents the out-of-plane coordinate. Under plane stress conditions, we have $\sigma_{33}=\sigma_{32}=\sigma_{31}=0$ or $\sigma_{33}=\sigma_{3 r}=\sigma_{3 \theta}=0$. Therefore, the yield or phase transformation criterion can be expressed in the Cartesian coordinates as

$$
f\left(\sigma_{i j}\right)=\left(\sigma_{11}^{2}+\sigma_{22}^{2}-\sigma_{11} \sigma_{22}+3 \sigma_{12}^{2}\right)^{1 / 2}+\frac{\mu}{\sqrt{3}}\left(\sigma_{11}+\sigma_{22}\right)-\sigma_{0}=0
$$

or in the polar coordinates as

$$
f\left(\sigma_{i j}\right)=\left(\sigma_{r r}^{2}+\sigma_{\theta \theta}^{2}-\sigma_{r r} \sigma_{\theta \theta}+3 \sigma_{r \theta}^{2}\right)^{1 / 2}+\frac{\mu}{\sqrt{3}}\left(\sigma_{r r}+\sigma_{\theta \theta}\right)-\sigma_{0}=0
$$

Further discussions on pressure-sensitive yielding can be found in Rudnicki and Rice [36] and Needleman and Rice [37]. Detailed information on a deformation plasticity version of the constitutive law can be found in Li and Pan [38]. 


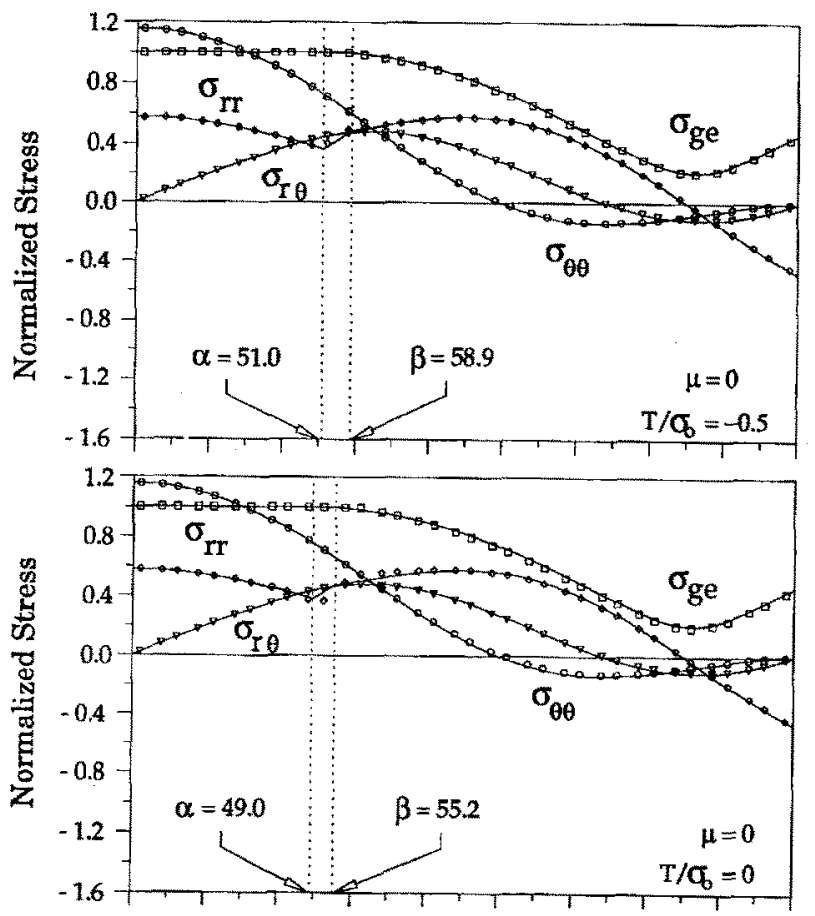

$$
\begin{aligned}
& \mu=0 \\
& \mathrm{~T} / \sigma_{0}=-0.5
\end{aligned}
$$

C.S.
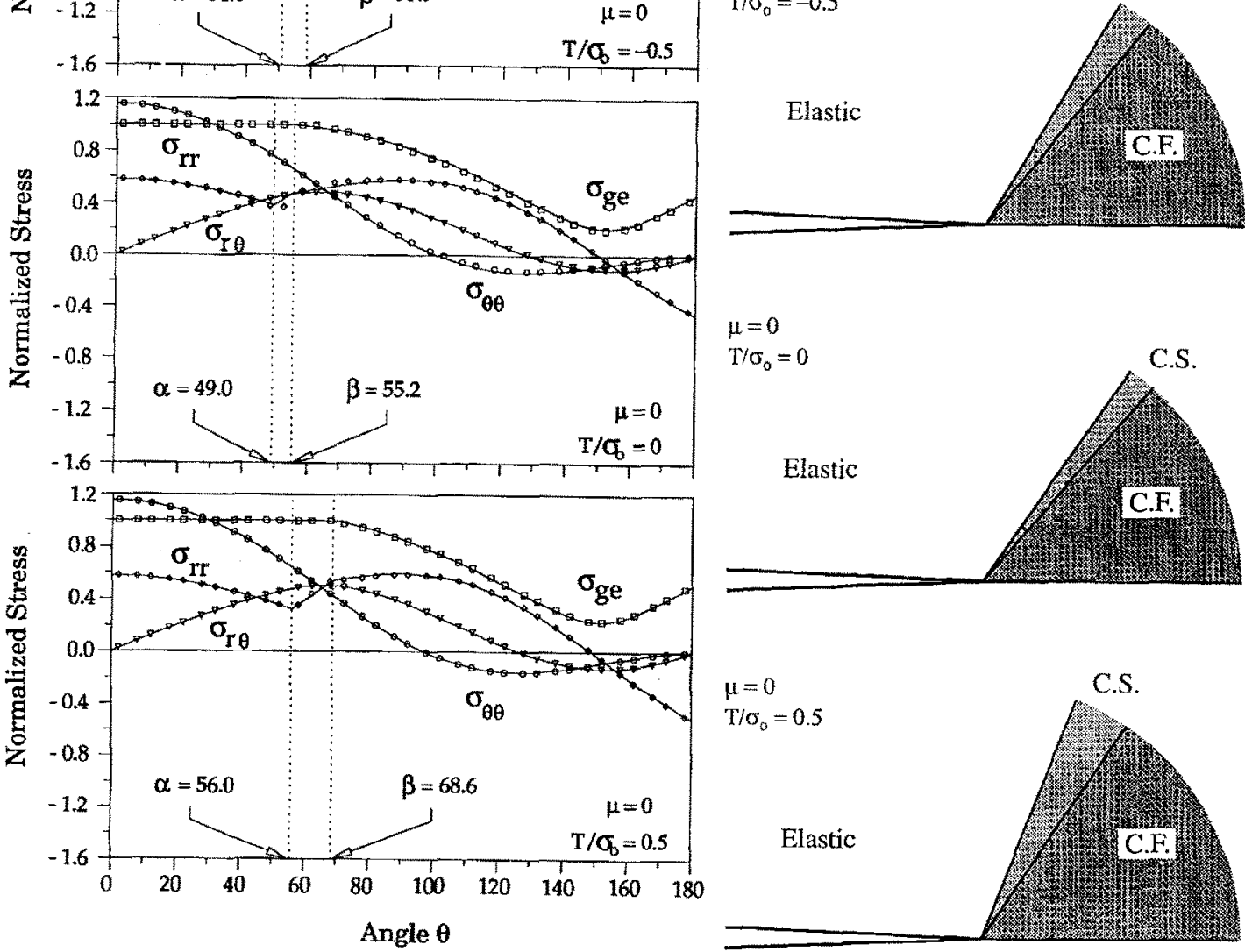

$\mu=0$

$T / \sigma_{0}=0$

C.S.

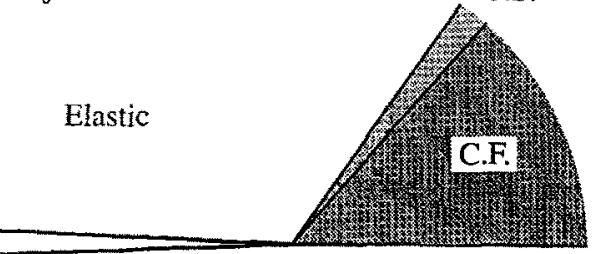

$\mu=0$

$\mathrm{T} / \sigma_{0}=0.5$

C.S.

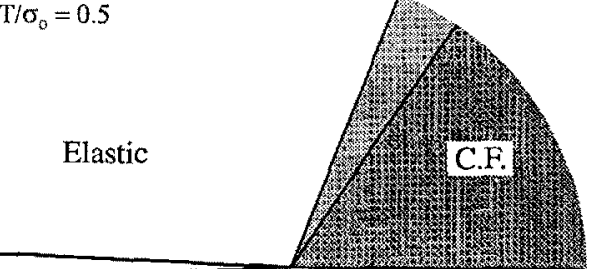

Figure 4. The angular variations of the crack-tip stresses nor- Figure 5 . The asymptotic crack-tip structures for malized by $\sigma_{0}$ at $r=2 J / \sigma_{0}$ for $\mu=0$. Top: $T / \sigma_{0}=-0.5$, elastic perfectly plastic materials with $\mu=0$. Center: $T / \sigma_{0}=0$, Bottom: $T / \sigma_{0}=0.5$. Top: $T / \sigma_{0}=-0.5$, Center: $T / \sigma_{0}=0$, Bottom: $T / \sigma_{0}=0.5$.

For Ce-TZP ceramics, the phase transformation gives an initial perfectly plastic response [39]. When both the available effective and volumetric phase transformation strains are much larger than the effective strain and the volumetric strain at the inception of the phase transformation, we have the so-called strong transformation case [29]. In this case, the phase transformation zone size and shape may possibly be close to those of the computational results for elastic perfectly plastic materials. Therefore we first investigate the near-tip fields and plastic zones for elastic perfectly plastic materials. It should be noted that the increased toughness due to phase transformation can be related to the height of the phase transformation zone [28]. 


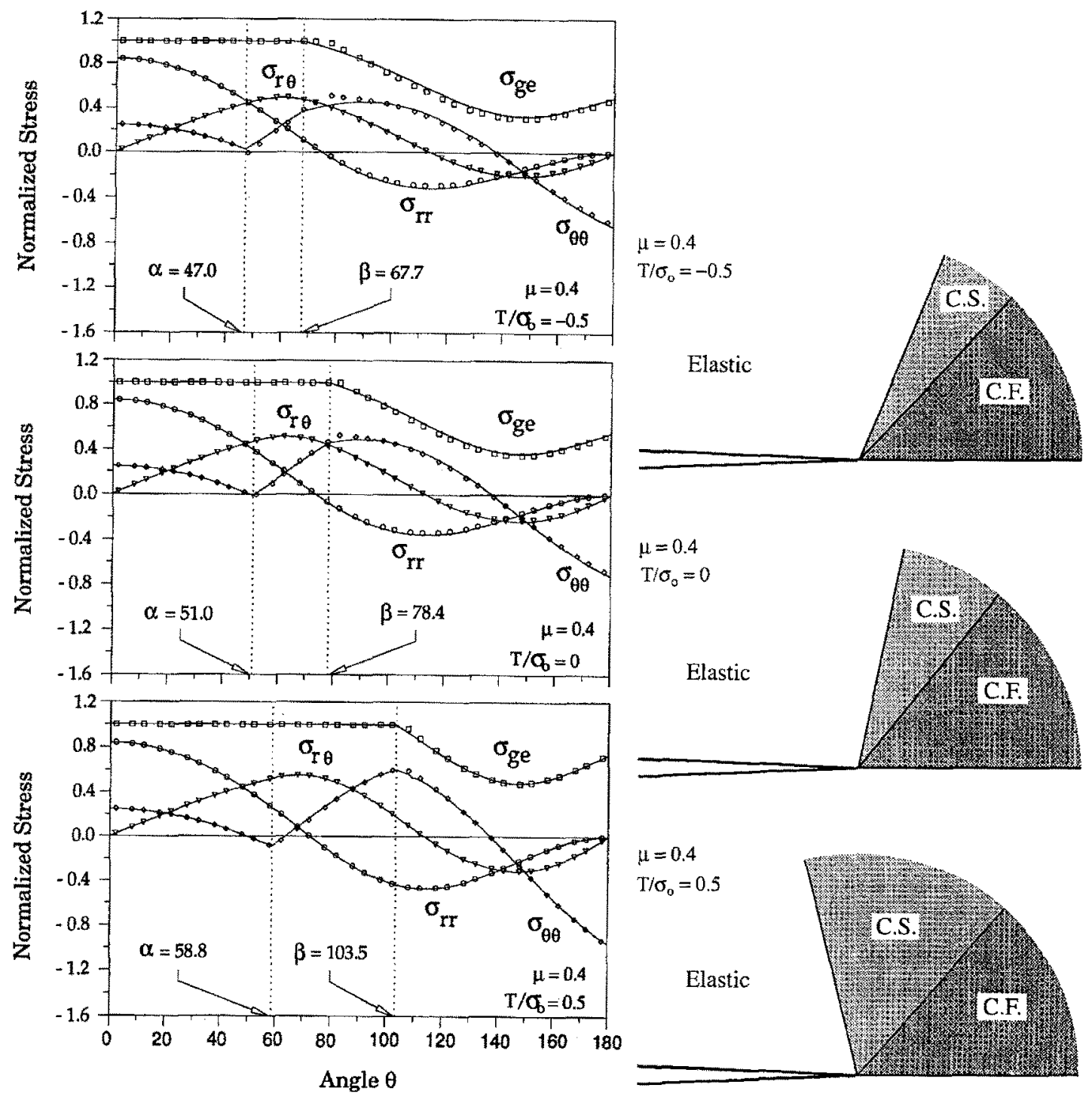

Figure 6 . The angular variations of the crack-tip stresses nor- Figure 7 . The asymptotic crack-tip structures for malized by $\sigma_{0}$ at $r=2 J / \sigma_{0}$ for $\mu=0.4$. Top: $T / \sigma_{0}=-0.5$. elastic perfectly plastic materials with $\mu=0.4$. Center: $T / \sigma_{0}=0$. Bottom: $T / \sigma_{0}=0.5$. Top: $T / \sigma_{0}=-0.5$. Center: $T / \sigma_{0}=0$. Bottom: $T / \sigma_{0}=0.5$.

In the experiment of Yu and Shetty [22], an elongated shape of the phase transformation zone with a sharp front was shown ahead of a crack tip in Ce-TZP. It cannot be explained by using the unperturbed linear elastic asymptotic crack-tip stress field and the Coulomb phase transformation criterion. The main reason for this disagreement could be that the phase transformation in these ceramics is strong and therefore using the elastic asymptotic solution is inappropriate. Therefore we here consider the large amount of phase transformation from the plasticity viewpoint and the constraint condition experienced near the crack tip using the two- 

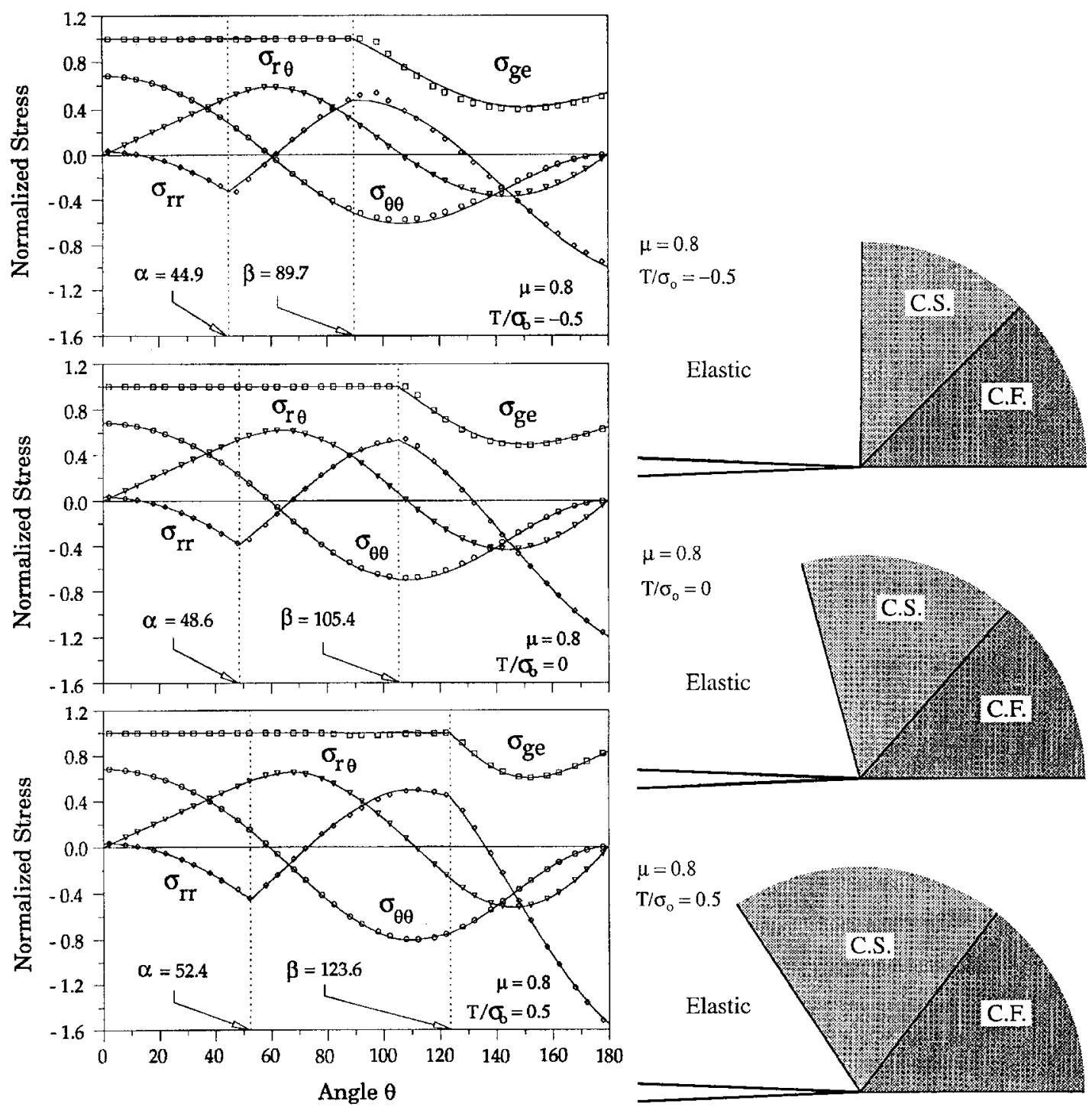

Figure 8. The angular variations of the crack-tip stresses nor- Figure 9. The asymptotic crack-tip structures for malized by $\sigma_{0}$ at $r=2 J / \sigma_{0}$ for $\mu=0.8$. Top: $T / \sigma_{0}=-0.5$. elastic perfectly plastic materials with $\mu=0.8$. Center: $T / \sigma_{()}=0$. Bottom: $T / \sigma_{0}=0.5$. Top: $T / \sigma_{0}=-0.5$. Center: $T / \sigma_{0}=0$. Bottom: $T / \sigma_{0}=0.5$.

parameter ( $K$ and $T$ ) boundary layer formulation from the fracture mechanics viewpoint. We also consider the finite plastic (phase transformation) strains that occurred in these ceramics.

In Figure 3, the generalized effective stress $\sigma_{g e}$ is shown as a function of the effective plastic (phase transformation) strain $\varepsilon_{e}\left(=\int\left(\frac{2}{3} \dot{\varepsilon}_{i j}^{p} \dot{\varepsilon}_{i j}^{p}\right)^{1 / 2} \mathrm{~d} t\right)$ accumulated throughout the deformation history. As shown in the figure, for perfectly plastic materials the effective plastic strain can be infinite. However, for transformation toughened ceramics, only a finite amount of the phase transformation effective strain $\varepsilon_{e}^{T}$ is available. After the exhaustion of the effective phase transformation strain, the material becomes elastic again. The effective and volumetric plastic 


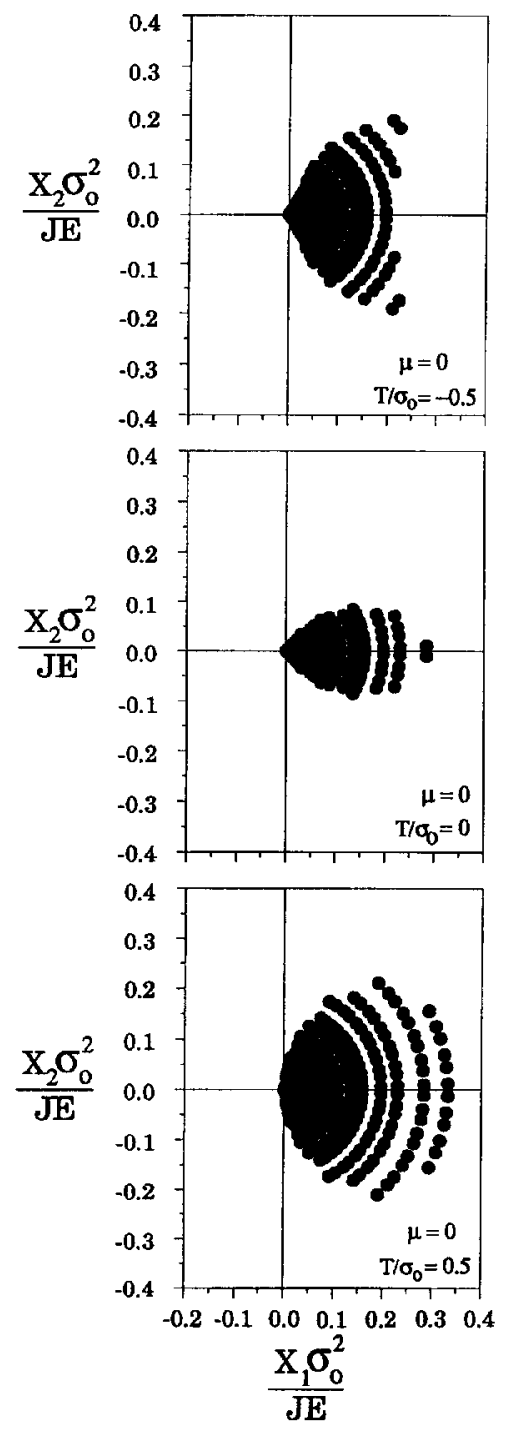

Figure 10. The normalized plastic zones from the finite element computations for perfectly plastic materials with $\mu=0$. Top: $T / \sigma_{0}=$ -0.5. Center: $T / \sigma_{0}=0$. Bottom: $T / \sigma_{0}=0.5$.
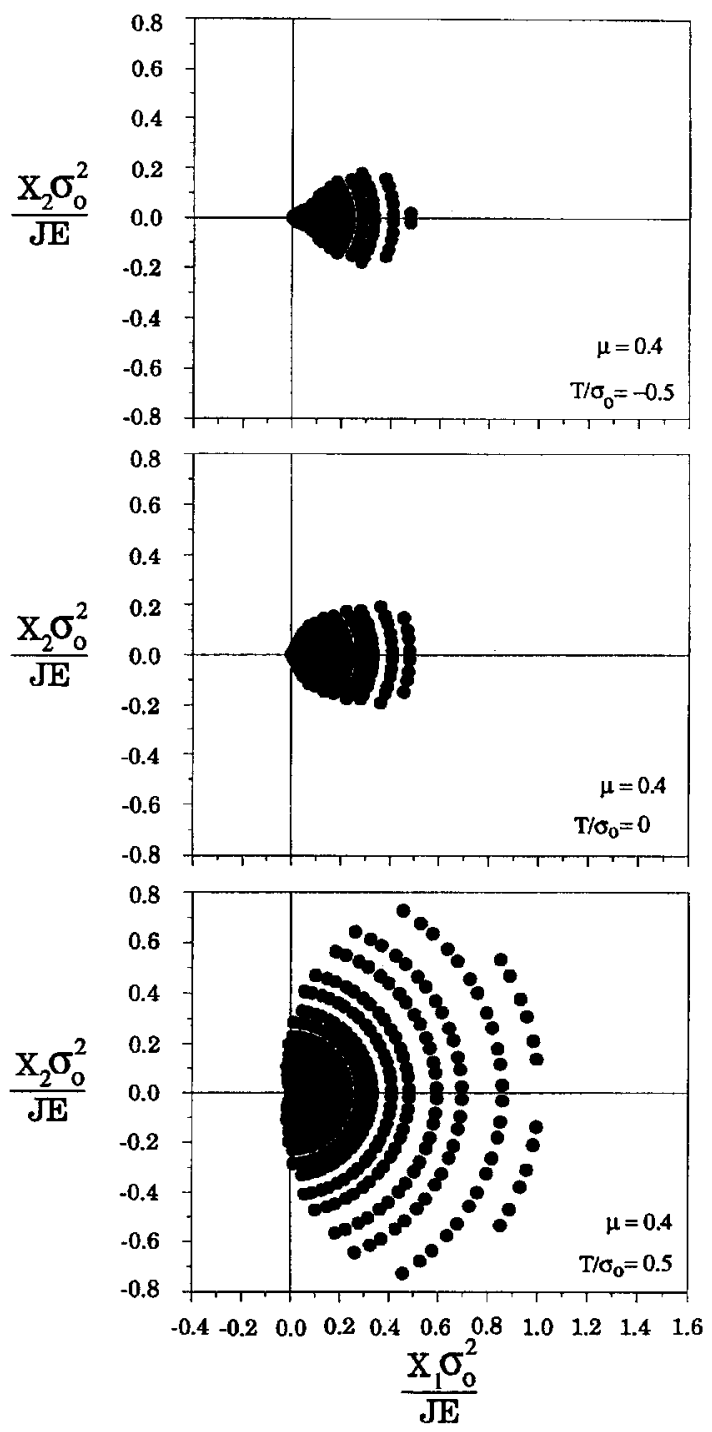

Figure 11. The normalized plastic zones from the finite element computations for perfectly plastic materials with $\mu=0.4$. Top: $T / \sigma_{0}=-0.5$. Center: $T / \sigma_{0}=0$. Bottom: $T / \sigma_{0}=0.5$.

(phase transformation) strain increments are related by a plastic dilatancy factor. Since the normality flow rule is assumed here, the pressure sensitivity factor equals the plastic dilatancy factor. A detailed description of the constitutive law with the normality flow rule for the plastic strain rate can be found in Dong and Pan [40].

\section{Finite element model}

We consider a crack in a circular domain of radius $r_{0}$. Due to the symmetry of mode I loading, we select the upper half of the circular domain as shown in Figure 2. The semi-circular domain 

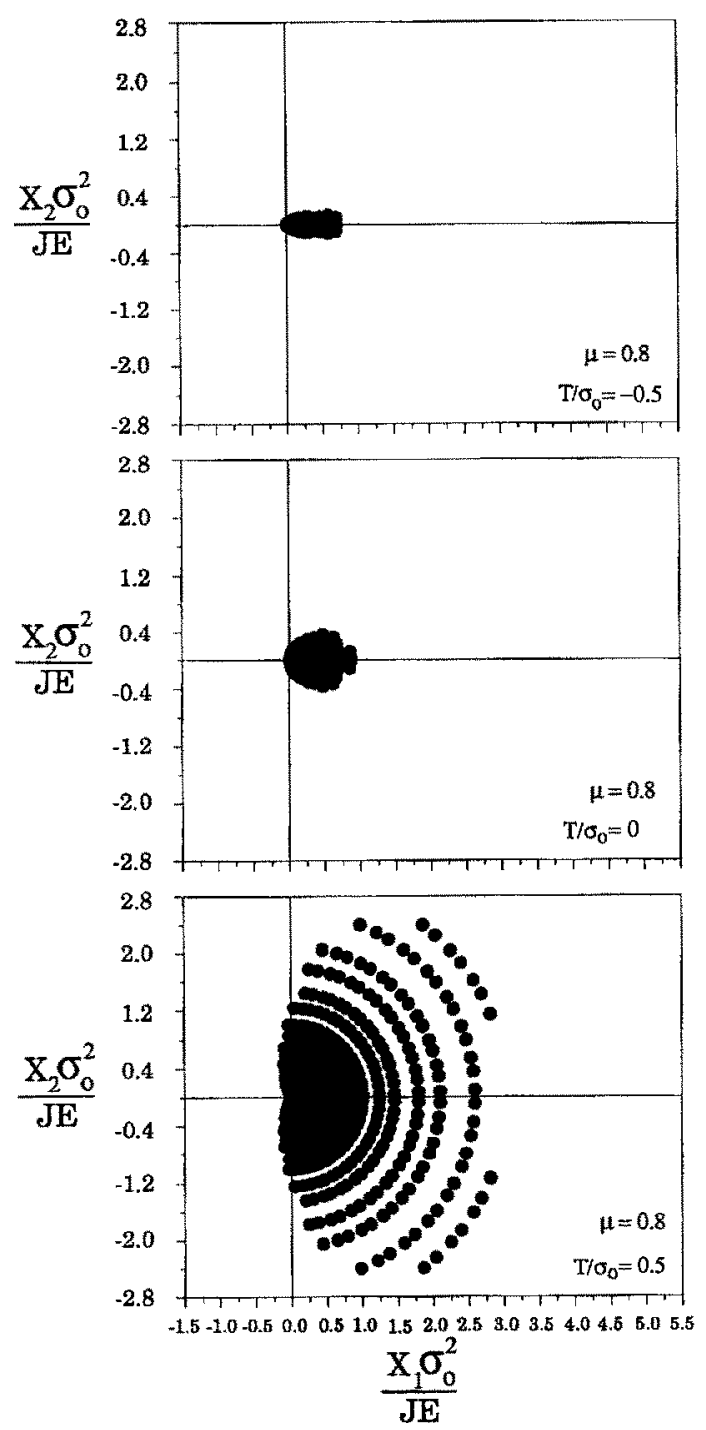

Figure 12. The normalized plastic zones from the finite element computations for perfectly plastic materials with $\mu=0.8$. Top: $T / \sigma_{0}=$ -0.5. Center: $T / \sigma_{0}=0$. Bottom: $T / \sigma_{0}=0.5$.
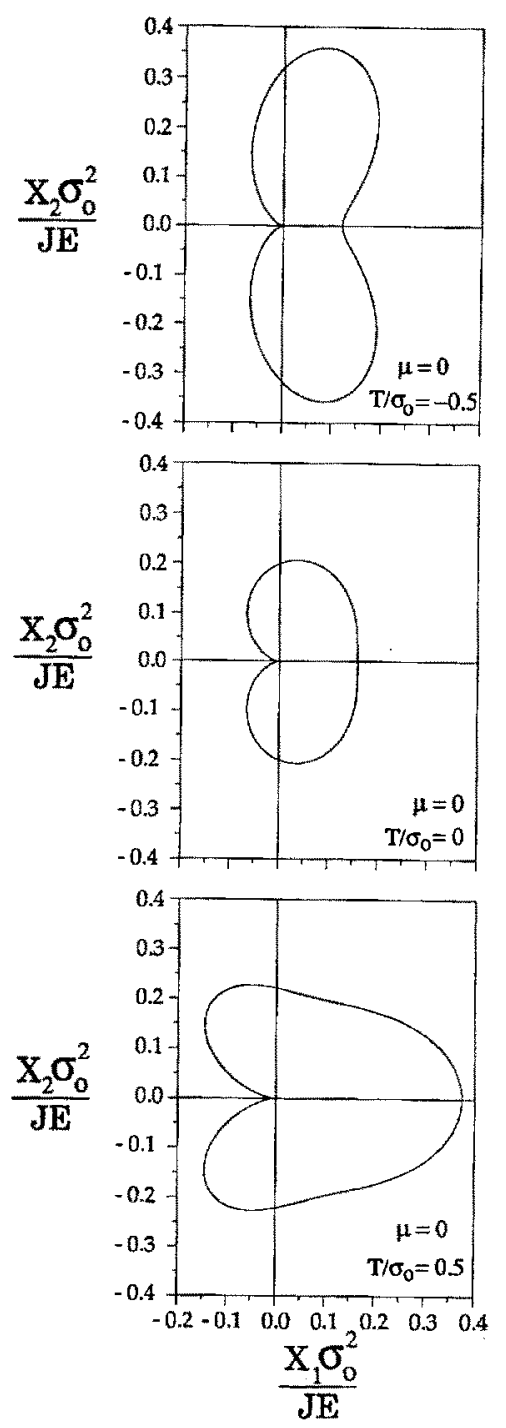

Figure 13. The normalized plastic zones from the linear elastic asymptotic stress fields for $\mu=0$. Top: $T / \sigma_{0}=-0.5$. Center: $T / \sigma_{0}=0$. Bottom: $T / \sigma_{0}=0.5$.

is entirely discretized by a mesh of 8-node serendipity quadrilateral elements. In the immediate crack-tip region, we use a ring of 18 wedge-shaped elements of size $r_{i}$ and we choose a ratio of $r_{i}$ to $r_{0}$ of $10^{-5}\left(r_{i} / r_{0}=10^{-5}\right)$. Elements in the $\theta$ direction are equally distributed from 0 to $\pi$. The wedge-shaped elements are surrounded by 24 semi-circular strips generated by a logarithmic scale in the radial direction. Therefore, there is a total of 450 elements in the mesh. For the wedge-shaped elements, the collapsed nodes technique is employed to simulate the $1 / r$ singularity needed for the strains in the plastic sectors near the tip. 

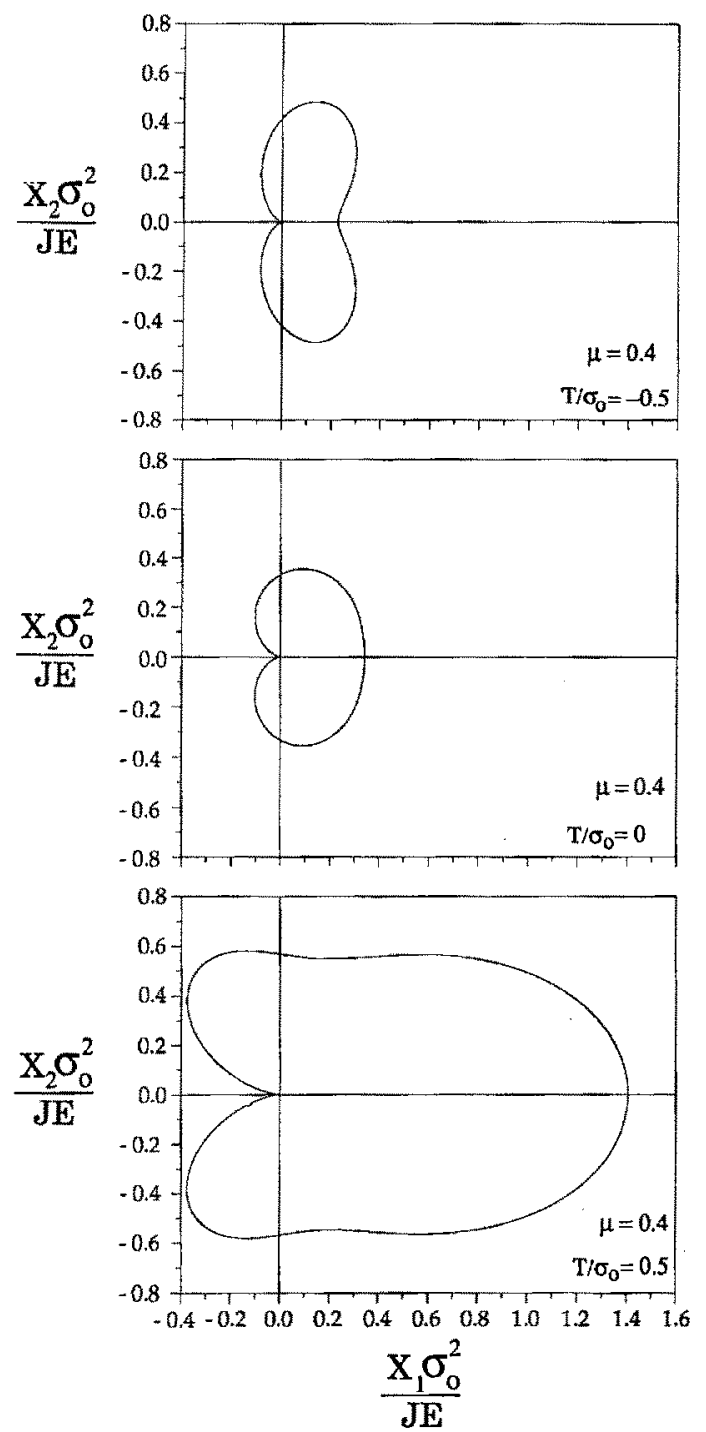

Figure 14. The normalized plastic zones from the linear elastic asymptotic stress fields for $\mu=0.4$. Top: $T / \sigma_{0}=-0.5$. Center: $T / \sigma_{0}=$ 0 . Bottom: $T / \sigma_{0}=0.5$.
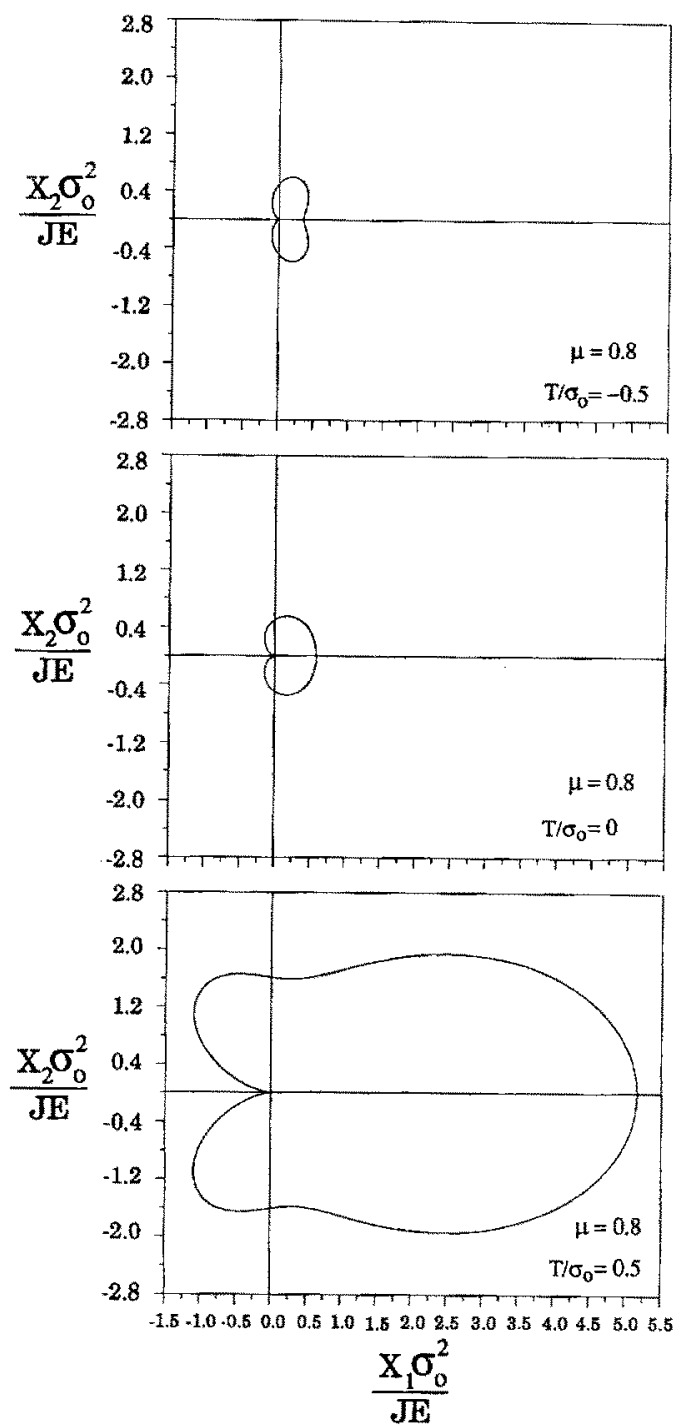

Figure 15. The normalized plastic zones from the linear elastic asymptotic stress fields for $\mu=0.8$. Top: $T / \sigma_{0}=-0.5$. Center: $T / \sigma_{0}=0$. Bottom; $T / \sigma_{0}=0.5$.

In the classical linear elastic fracture mechanics, the near-tip fields are assumed to be governed by the far-field mode I stress intensity factor $K$. Consequently, the small-scale yielding problem is replaced by a boundary layer formulation with the boundary condition along the circumference of the domain prescribed as

$$
\sigma_{i j}=\frac{\Pi}{\sqrt{2 \pi r}} f_{i j}(\theta)
$$



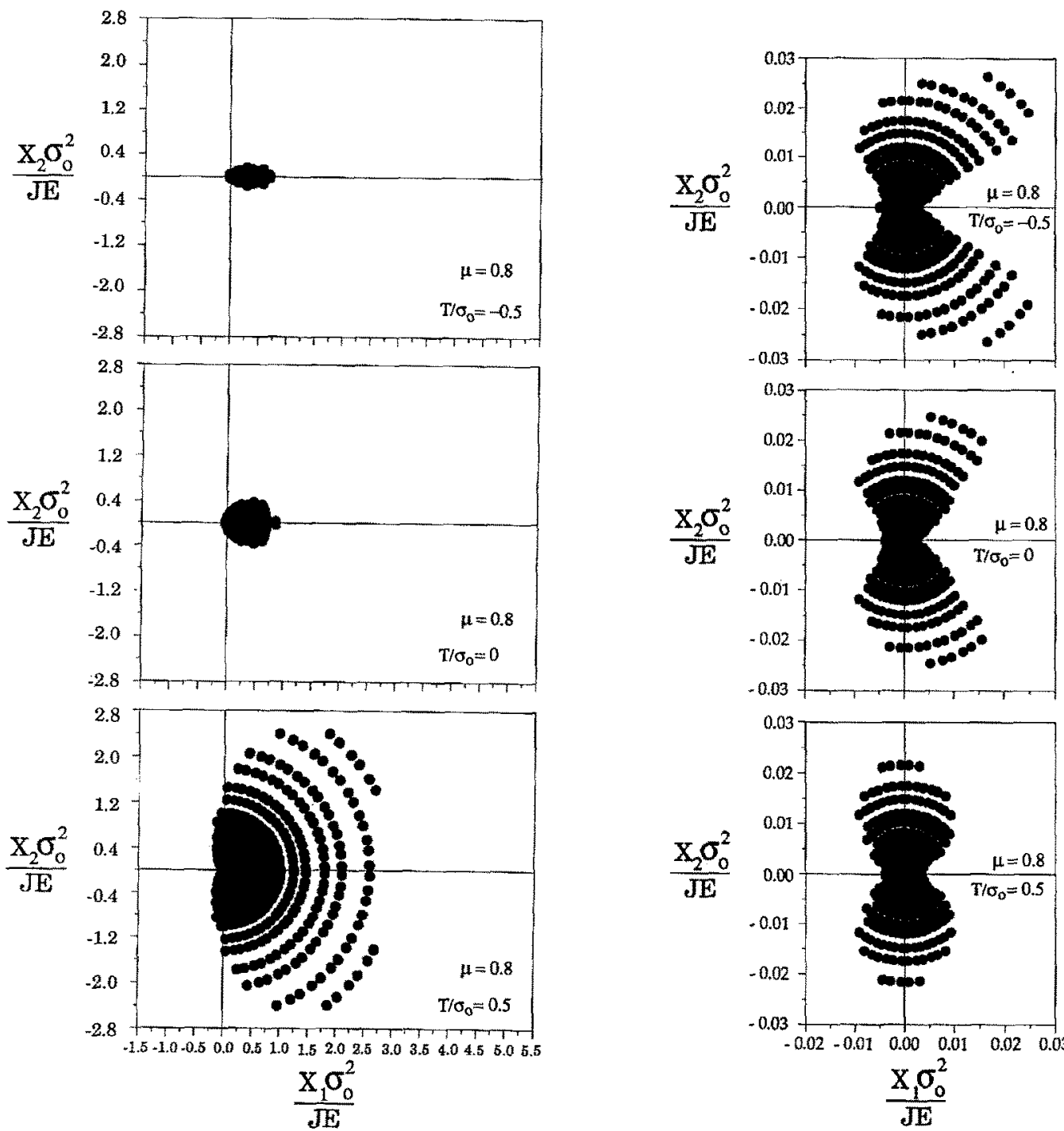

Figure 16. The normalized phase transformation zones for $\mu=0.8$. Top: $T / \sigma_{0}=-0.5$. Center: $T / \sigma_{0}=0$. Bottom: $T / \sigma_{0}=0.5$.

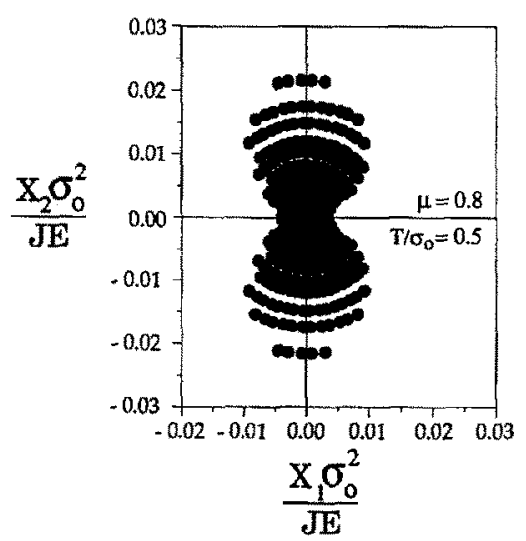

Figure 17. The normalized fully phase transformation zones for $\mu=0.8$. Top: $T / \sigma_{0}=-0.5$. Center: $T / \sigma_{0}=0$. Bottom: $T / \sigma_{0}=0.5$.

where $\sigma_{i j}$ represent the linear elastic asymptotic crack-tip stresses, and $f_{i j}(\theta)$ are dimensionless functions given by the linear elastic asymptotic solution (for example, see Kanninen and Popelar [41]). The familiar form in (4) is the leading term of the asymptotic crack-tip stress field derived by Williams [42] and Irwin [43].

When we include the $T$ stress in the asymptotic crack-tip stress solution, the boundary condition along the circumference of the domain becomes

$$
\sigma_{i j}=\frac{K}{\sqrt{2 \pi r}} f_{i j}(\theta)+T \delta_{1 i} \delta_{1 j}
$$



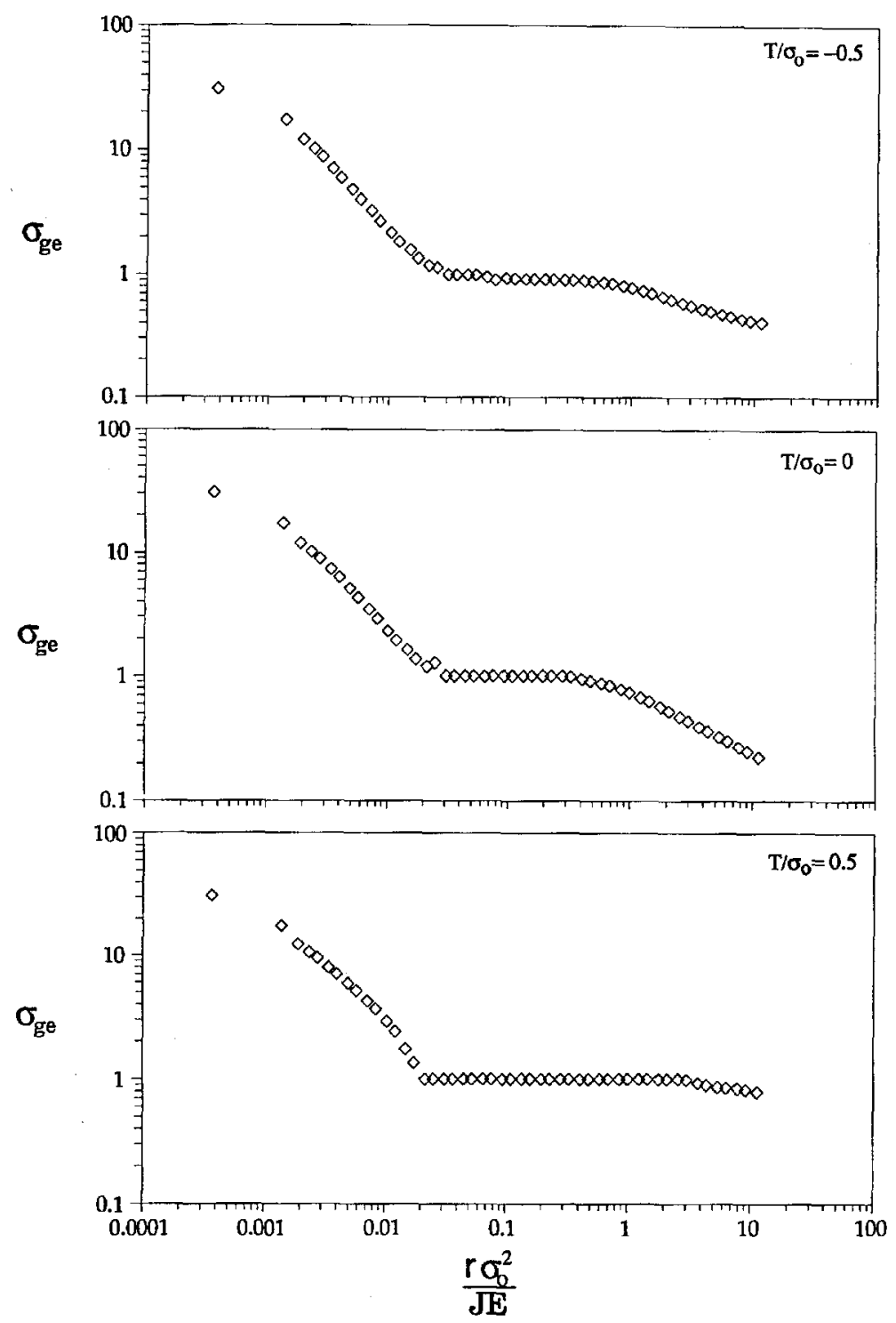

Figure 18. The generalized effective stress normalized by $\sigma_{0}$ at $\theta=62^{\circ}$ as a function of the radial distance normalized by $J E / \sigma_{0}^{2}$ for $\mu=0.8$. Top: $T / \sigma_{0}=-0.5$. Center: $T / \sigma_{0}=0$. Bottom: $T / \sigma_{0}=0.5$.

where $T$ is the normal stress parallel to the crack faces. The magnitude of the $T$ stress is defined through a biaxiality parameter $B$ introduced by Leevers and Radon [44]

$$
B=\frac{T \sqrt{\pi a}}{K}
$$

When (5) and (6) are combined, we can see that the stress field is scaled by the magnitude of $K$ for a given $B$. Therefore a proportional loading condition is maintained along the circumferential boundary as we monotonically increase the magnitude of $K$. 

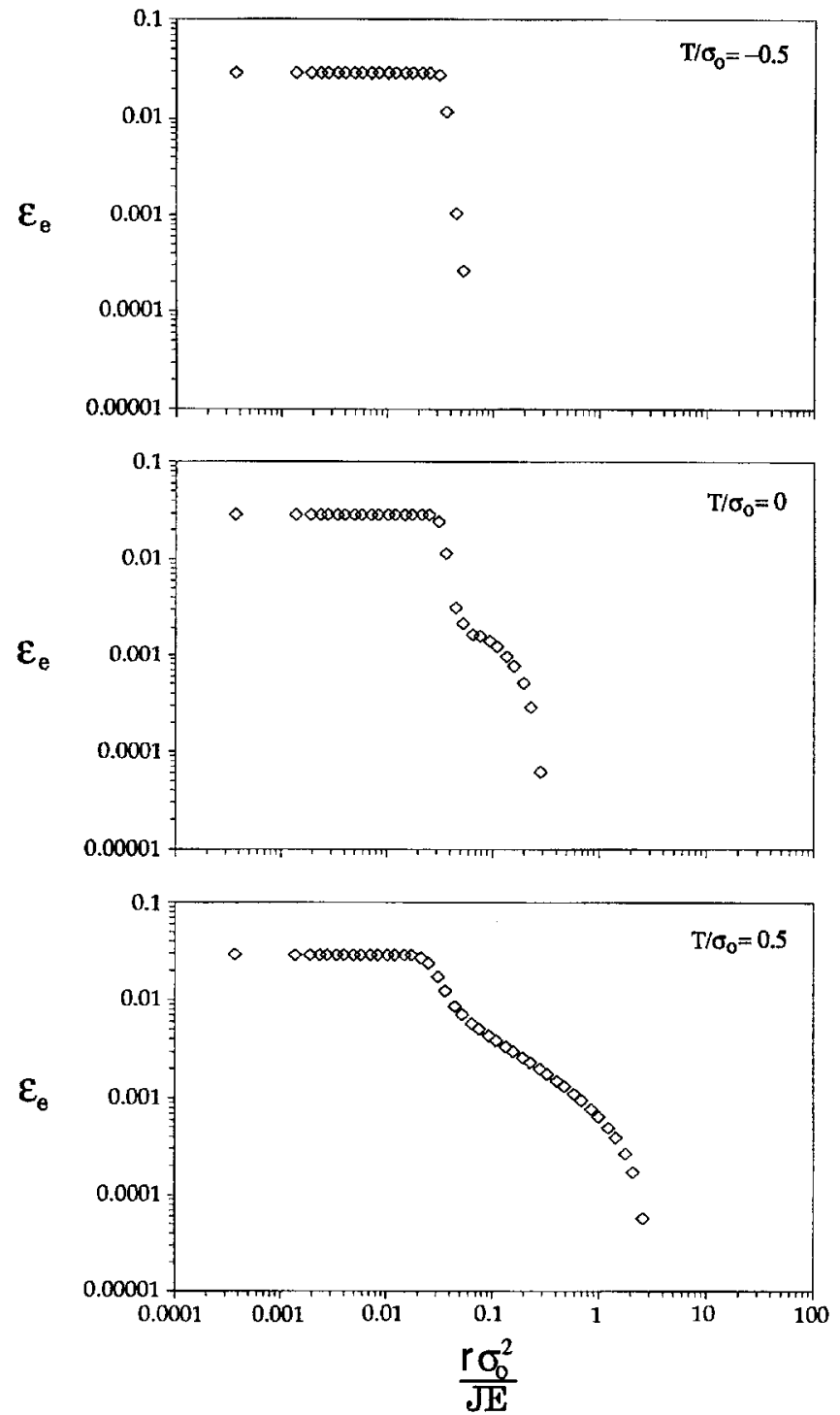

Figure 19. The effective phase transformation strain at $\theta=62^{\circ}$ as a function of the radial distance normalized by $J E / \sigma_{0}^{2}$ for $\mu=0.8$. Top: $T / \sigma_{0}=-0.5$. Center: $T / \sigma_{0}=0$. Bottom: $T / \sigma_{0}=0.5$.

In this study the loading is applied through the in-plane displacements $u_{i}$ along the circumferential boundary of the semi-circular domain as

$$
u_{i}=\frac{K}{2 G}\left(\frac{r_{0}}{2 \pi}\right)^{1 / 2} \tilde{u}_{i}(\theta, \nu)+\frac{T}{E} r_{0} \hat{u}_{i}(\theta, \nu),
$$

where $E$ and $G$ represent Young's modulus and shear modulus, respectively. The dimensionless functions $\tilde{u}_{i}(\theta, \nu)$ represent the linear elastic asymptotic crack-tip displacements as functions of $\theta$ and Poisson's ratio $\nu$ (for example, see [41]). The dimensionless functions $\hat{u}_{i}(\theta, \nu)$ represent the solutions as functions of $\theta$ and $\nu$ due to the non-singular stress $T$. 


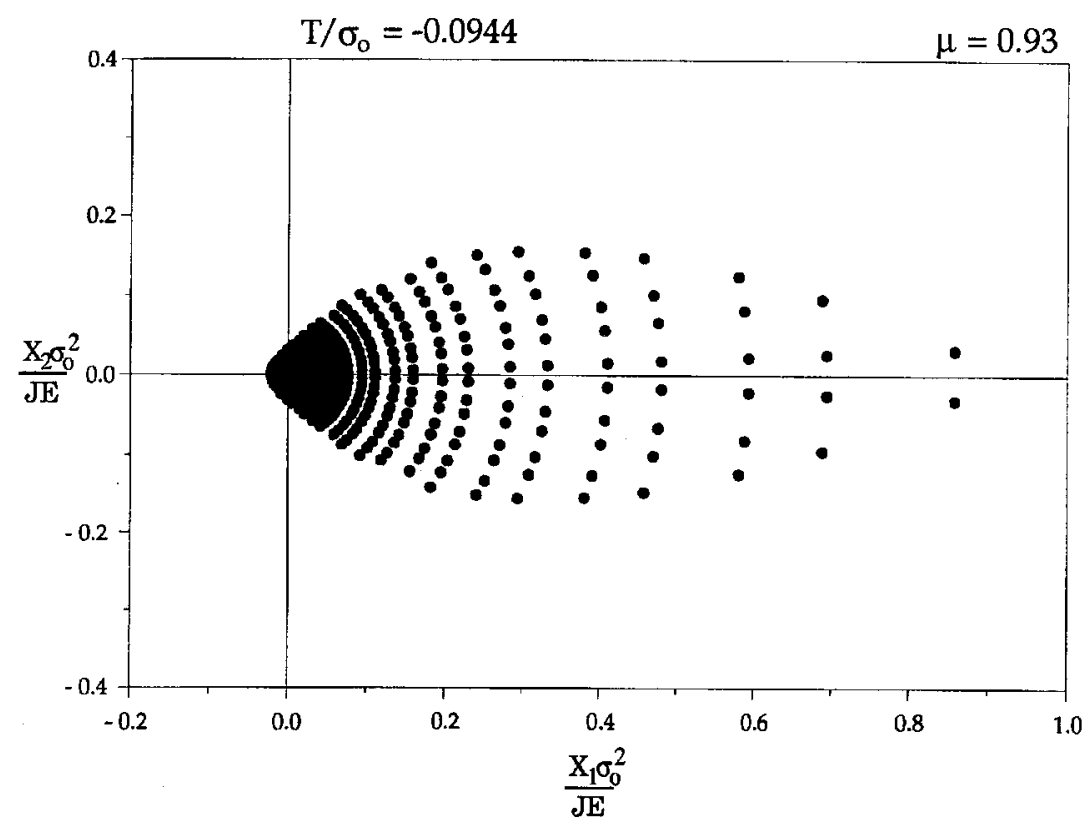

Figure 20. The normalized phase transformation zone for $\mu=0.93$ and $T / \sigma_{0}=-0.0944$.

\section{Numerical results for perfectly plastic materials}

Finite element computations were performed for $\mu=0$ (for pressure-insensitive Mises materials), 0.4 , and 0.8 . These values of $\mu$ were chosen to encompass a full range of engineering materials. In all the results reported in the following, the ratio of Young's modulus $E$ to the generalized tensile effective stress $\sigma_{0}$ was taken as 500, and Poisson's ratio $\nu$ was taken as 0.3 .

The sampling Gauss points for our stress results are chosen at a radial distance of $2 J / \sigma_{0}$, or equivalently $2 K^{2} /\left(E \sigma_{0}\right)$ [16]. This sampling radius was chosen so that the integration points are well inside the plastic zone, to give a good approximation to the near-tip stresses, and away from the effects of the crack blunting. A detailed study on crack-tip blunting can be found in McMeeking [45].

The numerical results are compared with those of the corresponding asymptotic analyses based on the research work of Ben Aoun and Pan [21]. It should be briefly mentioned that there are three types of sectors for the near-tip fields under plane stress conditions. These are constant stress (C.S.) sector, centered fan (C.F.) sector, and elastic sector. The assembly of the sectors based on the corresponding finite element solutions is detailed in [21].

The crack-tip stress fields for $\mu=0$ are shown in Figures 4 and 5. The angular distribution of the stresses in the polar coordinates are plotted in Figure 4 where the top figure is for $T / \sigma_{0}=-0.5$, the middle one is for $T / \sigma_{0}=0$, and the bottom figure is for $T / \sigma_{0}=0.5$. In these plots, the symbols represent the stresses of the Gauss points which form a fan surrounding the crack tip, and the solid lines represent the stresses evaluated by the asymptotic analysis detailed in [21]. All the stresses are normalized by the reference stress $\sigma_{0}$. Figure 5 shows a schematic distribution of the crack-tip sectors for various $T$ stresses. In these plots, C.S. represents a constant stress sector, C.F. represents a centered fan sector, and Elastic represents an elastic sector. Similar results for $\mu=0.4$ are plotted in Figures 6 and 7, and the results for $\mu=0.8$ are plotted in Figures 8 and 9. 
In these figures, we can see that the finite element solutions (symbols) agree well with the corresponding asymptotic solutions (solid lines). The excellent agreement attests to the accuracy of our finite element computations and the corresponding asymptotic solutions. It is also important to note that all the stress components including $\sigma_{r r}$ are continuous in the $\theta$ direction in contrast to the existence of the radial stress discontinuity in the asymptotic solutions where the material surrounding the crack tip is assumed to be fully yielded in [5] and [20]. When the crack-tip stresses from our computations are plotted in the Cartesian coordinates, the stresses appear to be constant in the constant stress sectors.

As shown in Figure 6 for $\mu=0.4$, when the $T$ stress increases, the angular span of the centered fan sector increases slightly, from $47^{\circ}$ for $T / \sigma_{0}=-0.5$, to $51^{\circ}$ for $T / \sigma_{0}=0$, and to $58.8^{\circ}$ for $T / \sigma_{0}=0.5$. This effect is more pronounced for the angular span of the constant stress sector, which is $20.7^{\circ}$ for $T / \sigma_{0}=-0.5,27.4^{\circ}$ for $T / \sigma_{0}=0$, and $44.7^{\circ}$ for $T / \sigma_{0}=0.5$. Adding these two effects, we can see that the angular span of the plastic sectors increases for increasing $T$ stress. This behavior was consistently observed for $\mu=0.8$. However, this trend is not observed for the case of $\mu=0$ where $T / \sigma_{0}=-0.5$ results in a slight increase of the angular span of the plastic sectors when compared with that of $T / \sigma_{0}=0$. Therefore it is reasonable to expect that for $\mu$ close to 0 , the trend should be close to that of $\mu=0$. Nevertheless, for a moderately large $\mu$, the angular span of the plastic sectors increases as the $T$ stress increases. It should be mentioned that for $T / \sigma_{0}=0$, the angular spans of the plastic sectors at different $\mu$ 's are slightly different from those in [21]. The reason is that the sampling Gauss points for the angular variation of the stresses are different in the two investigations. In this study the sampling Gauss points are located at $r=2 J / \sigma_{0}$ whereas the sampling Gauss points in [21] are located at $r / r_{p} \approx 10^{-2}$ where $r_{p}$ represents the plastic zone size at $\theta=0$.

The plastic zones from the finite element solutions, normalized by $J E / \sigma_{0}^{2}$ (or $K^{2} / \sigma_{0}^{2}$ ), are shown in Figures 10,11 and 12 for $\mu=0,0.4$, and 0.8 , respectively. The dots in these plots represent the Gauss points in their plastic state. The normalized plastic zones based on the linear elastic asymptotic stress fields under plane stress conditions for $\mu=0,0.4$, and 0.8 are plotted in Figures 13, 14, and 15, respectively. In these figures, we can clearly see that the $T$ stress has significant effects on the size and shape of the plastic zone for both pressure-insensitive and pressure-sensitive materials. In Figure 12 for a large pressure sensitivity of $\mu=0.8$, the plastic zone height increases substantially when $T$ increases. As shown in Figure 15 for $\mu=0.8$ the linear elastic asymptotic stress fields give a similar trend on the plastic zone height as a function of the $T$ stress. However, the linear elastic asymptotic stress field cannot give the plastic zone of a long narrow shape as in Figure 12 for elastic perfectly plastic materials at $T / \sigma_{0}=-0.5$. In summary, full-field finite element computations are needed to accurately characterize the heights and shapes of the plastic zones for perfectly plastic materials. The plastic zone solutions based on the linear elastic asymptotic stress fields, however, can be used as good approximations for weak transformation materials where the available phase transformation strains are much smaller than the effective strain and the volumetric strain at the inception of the phase transformation.

For perfectly plastic materials under plane stress conditions, a centered fan sector is located ahead of the tip. The stresses in the centered fan sector are fully determined [21]. Therefore the $T$ stress has no influence on the stresses and hydrostatic tension directly ahead of the tip. However, the $T$ stress can affect the angular spans of the centered fan sector, the neighboring constant stress sector and the elastic sector bordering the crack face as well as the sizes and shapes of plastic zones. On the other hand, for perfectly plastic materials under plane strain conditions, a constant stress sector is located ahead of the tip. The angular span of the front 
constant stress sector is determined for a given pressure sensitivity [15]. Therefore the $T$ stress has no influence on the angular span of the front constant stress sector. The $T$ stress, however, affects the angular spans of the other crack-tip sectors: a centered fan sector, another constant stress sector and an elastic sector. It turns out that the $T$ stress has significant effects on the stresses and hydrostatic tension directly ahead of the tip for materials with small pressure sensitivity. However, as the pressure sensitivity increases, the effects on the stresses and hydrostatic tension directly ahead of the tip decreases, as shown in [15]. In general, when the value of $T$ decreases, the stresses and hydrostatic tension directly ahead of the tip decrease. The $T$ stress can also affect the sizes and shapes of plastic zones as shown in [15] under plane strain conditions.

\section{Numerical results for phase transformation materials}

Chen [27] reported that $\mu=0.55$ for Mg-PSZ and $\mu=0.77$ for Ce-TZP. Yu and Shetty [22] reported that $\mu=0.93$ for Ce-TZP. Because of the high computational cost due to the introduction of the second elastic behavior after the exhaustion of the phase transformation strains, we only investigate the case with a large pressure sensitivity of $\mu=0.8$. The results will be used to compare with those of $\mu=0.8$ for perfectly plastic materials. The available effective plastic (phase transformation) strain $\varepsilon_{e}$ is taken to be 0.029 which is related to the volumetric phase transformation strain of 0.04 by the normality flow rule for $\mu=0.8$.

The normalized phase transformation zones for $\mu=0.8$ are given in Figure 16 for various $T$ stress values. The phase transformation zone is defined where the phase transformation criterion is met and/or the phase transformation strains are not zero. In these plots, the phase transformation zones are normalized by $J E / \sigma_{0}^{2}$ (or $K^{2} / \sigma_{0}^{2}$ ) in the $x_{1}$ - and $x_{2}$-directions. The dimensions in both directions are kept to the same scale to illustrate the actual geometry of the transformation zones. In the top plot of Figure 16 for $T / \sigma_{0}=-0.5$, we note that the plastic zone is narrow and concentrated in front of the crack. In the bottom plot of Figure 16 for $T / \sigma_{0}=0.5$, the phase transformation zone becomes wider. The height of the phase transformation zone increases substantially as $T / \sigma_{0}$ increases from -0.5 to 0.5 .

When we compare Figure 16 for phase transformation materials with Figure 12 for perfectly plastic materials, the phase transformation zone and the plastic zone are almost identical for a given $T / \sigma_{0}$. The only difference is that the front of the phase transformation zone appears to be sharper for phase transformation materials (note that two fronts appear for $T / \sigma_{0}=0.5$ ). This sharper front for the phase transformation materials can be thought of as the consequence of the second elastic response after the exhaustion of phase transformation strains. A similar trend of a sharper front of the phase transformation zone is also shown due to the second elastic response after the exhaustion of the phase transformation strains under plane strain conditions (Kim and Pan [46]).

The normalized fully transformed zones are plotted in Figure 17 for various $T$ stresses. The fully transformed zone is defined where the material is in the second elastic state after the exhaustion of the phase transformation strains (see Figure 3). In these plots, the phase transformation zones are normalized by $J E / \sigma_{0}^{2}$ (or $K^{2} / \sigma_{0}^{2}$ ) in the $x_{1}$ - and $x_{2}$-directions. The dimensions in both directions are kept to the same scale to illustrate the actual geometry of the fully transformed zones. We note that the heights of the fully transformed zones are relatively small when compared to those of the phase transformation zones for $T / \sigma_{0}=0$ and 0.5 . The fully transformed zone is leaning forward for $T / \sigma_{0}=-0.5$. It shifts toward the faces of the crack as $T$ increases. 
The radial variations of the generalized effective stress $\sigma_{g e}$ at $\theta=62^{\circ}$ are plotted in Figure 18 for various $T$ stresses. Note that in Figure 16 the angle $\theta_{\max }$, where the maximum height of the phase transformation zone occurs, varies approximately from $30^{\circ}$ to $60^{\circ}$ as $T / \sigma_{0}$ increases from -0.5 to 0.5 . If the phase transformation zone is assumed to be controlled by the hydrostatic stress only and the phase transformation zone shape is assumed to be based on the unperturbed linear elastic asymptotic crack-tip stress field, the maximum height occurs at $\theta=60^{\circ}$ [28]. Here the results at $\theta=62^{\circ}$ is used to compare the distribution of the phase transformation stress at this angle for the three cases with different values of $T / \sigma_{0}$. In these figures, the generalized effective stress is normalized by the reference stress $\sigma_{0}$ (at which the transformation occurs), and the radial distance is normalized by $J E / \sigma_{0}^{2}$, or equivalently $K^{2} / \sigma_{0}^{2}$. As shown in Figure 3, a fully transformed material element can be recognized by the value of the generalized effective stress which is larger than the reference stress $\sigma_{0}$. The transformation zone size in the $\theta=62^{\circ}$ direction therefore can be recognized by the extent of the flat region in these plots. As shown in Figure 18, the transformation zone size increases as $T$ increases.

The radial variations of the effective phase transformation strain $\varepsilon_{e}$ are plotted in Figure 19 for $\theta=62^{\circ}$ for various $T$ stresses. The flat regions in these plots are representative of the fully transformed zones. In the top plot of Figure 19 for $T / \sigma_{0}=-0.5$, we can see that most of the Gauss points where the phase transformation occurs are actually fully transformed (18 out of 21 phase transformed points are fully transformed). This demonstrates that, for the negative $T$ stress, the size of the phase transformation zone in the $\theta=62^{\circ}$ direction becomes close to that of the fully transformed zone.

As shown in [22], the phase transformation zone near a crack in ceria-partially-stabilized zirconia polycrystals $(\mu=0.93)$ appears to be a long narrow spear. This spear shape of the phase transformation zone cannot be explained by the analysis based on the unperturbed linear elastic asymptotic crack-tip stress field. Yu and Shetty used single-edge notch bend specimens with $w$ (width) $=15 \mathrm{~mm}, S$ (support span) $=60 \mathrm{~mm}$ and $a$ (precrack length) $=3.0$ to $3.5 \mathrm{~mm}$. For the zirconia ceramics, the available volumetric phase transformation strain is 0.04 and the generalized tensile effective stress $\sigma_{0}$ for phase transformation is $295.8 \mathrm{MPa}$. We take the biaxiality factor $B=-0.3$ for the single-edge notch bend specimen with $a / w=0.2$ from [44]. For the geometry and the loading of the specimen at $K=9.03 \mathrm{MPa}, T / \sigma_{0}$ is estimated to be -0.094 . In our calculation, we use an effective phase transformation strain $\varepsilon_{e}$ of 0.025 which is related to the available volumetric phase transformation strain of 0.04 by the normality flow rule for $\mu=0.93$.

Figure 20 shows the normalized phase transformation zone from our computation. Our computational result gives a transformation zone of a spear shape which has the length to height ratio of about 7. Yu and Shetty [22] reported the transformation zone of a spear shape with the length to height ratio of about 13 in their experiments. At least our computational result presented here gives a phase transformation of a spear shape which cannot be produced by the unperturbed linear elastic asymptotic stress field as presented in [22].

\section{Conclusion}

In this paper, we studied the effects of the $T$ stress on the plane-stress crack-tip fields for elasticplastic pressure-sensitive materials. For elastic perfectly plastic materials, good agreement between the computational results and the corresponding asymptotic solutions attest the accuracy of our numerical procedure. Due to the high computational cost, we only investigated 
the case with finite phase transformation strains for $\mu=0.8$. The computational results indicate the effects of the $T$ stress on the radial distribution of the phase transformation strains. The computational results also indicate that the sizes and the shapes of the phase transformation zones for strong phase transformation materials with large pressure sensitivity can be approximated by those for elastic perfectly plastic materials.

Finally we obtained the phase transformation zone based on the two-parameter boundary layer formulation to simulate the constraint condition experienced by the crack in the experiment of [22]. Our computational result shows a spear shape of the phase transformation zone which agrees well with the experimental observation. It should be noted that the spear shape of the phase transformation zone has not been explained previously. In this study, we at least identify three important factors that result in the spear shape of the phase transformation zone: the pressure and shear sensitivity of phase transformation, the finite phase transformation strain, and the constraint condition (the $T$ stress). Further modification of the phase transformation criterion at large hydrostatic stress and the hardening or softening behavior of the phase transformation may result in better agreement with the experimental results.

\section{Acknowledgment}

Z.E.A. Ben-Aoun acknowledges the financial support of the University Mission of Tunisia for its scholarship. Also, financial support of the work was provided by the National Science Foundation under grant number DMR-8708405.

\section{References}

1. S.G. Larsson and A.J. Carlsson, Journal of the Mechanics and Physics of Solids 21 (1973) $263-277$.

2. J.R. Rice, Journal of the Mechanics and Physics of Solids 22 (1974) 17-26.

3. J.R. Rice, Joumal of Applied Mechanics 35 (1968) 379-386.

4. J.W. Hutchinson, Journal of the Mechanics and Physics of Solids 16 (1968) 13-31.

5. J.W. Hutchinson, Journal of the Mechanics and Physics of Solids 16 (1968) 337-347.

6. J.R. Rice and G.F. Rosengren, Journal of the Mechanics and Physics of Solids 16 (1968) 1-12.

7. J.W. Hutchinson, Journal of Applied Mechanics 50 (1983) 1042-1051.

8. R.M. McMeeking and D.M. Parks, Elastic-Plastic Fracture Mechanics, ASTM STP 668, Philadelphia, Pa (1979) 175-194.

9. C.F. Shih and M.D. German, International Journal of Fracture 17 (1981) 27-43.

10. C. Betegón and J.W. Hancock, Journal of Applied Mechanics 58 (1991) 104-110.

11. A.M. Al-Ani and J.W. Hancock, Journal of the Mechanics and Physics of Solids 39 (1991) 23-43.

12. J.W. Hancock, W.G. Reuter and D.M. Parks, in Constraint Effects in Fracture, ASTM STP 1 171, Philadelphia, $\mathrm{Pa}$ (1993) 21-40.

13. Z.Z. Du and J.W. Hancock, Journal of the Mechanics and Physics of Solids 39 (1991) 555-567.

14. P. Dong and J. Pan, International Journal of Fracture 45 (1990) 243-262.

15. M. Kim and J. Pan, International Journal of Fracture 68 (1994) 1-34.

16. N.P. O'Dowd and C.F. Shih, Journal of the Mechanics and Physics of Solids 39 (1991) 989-1015.

17. Ibid., 40 (1992) 939-963.

18. J. Pan and C.F. Shih, International Journal of Fracture 37 (1988) 171-195.

19. J. Pan, International Journal of Fracture 38 (1988) 103-122.

20. F.Z. Li and J. Pan, Engineering Fracture Mechanics 35 (1990) 1105-1116.

21. Z.E.A. Ben-Aoun and J. Pan, Engineering Fracture Mechanics 44 (1993) 649-662.

22. C.-S. Yu and D. K. Shetty, Journal of the American Ceramic Society 72 (1989) 921-928.

23. D.C. Drucker and W. Prager, Quarterly of Applied Mathematics 10 (1952) 157-164.

24. W.A. Spitzig, R.J. Sober and O. Richmond, Acta Metallurgica 23 (1975) 885-893.

25. W.A. Spitzig, R.J. Sober and O. Richmond, Metallurgical Transactions 7A (1976) 1703-1710.

26. A.J. Kinloch and R.J. Young, Fracture Behaviour of Polymers, Elsevier Applied Science, London (1983).

27. I.-W. Chen, Journal of the American Ceramic Society 74 (1991) 2564-2572.

28. R.M. McMeeking and A.G. Evans, Journal of the American Ceramic Society 65 (1982) 242-246. 
29. B. Budiansky, J. W. Hutchinson and J. C. Lambropoulos, International Journal of Solids and Structures 19 (1983) $337-355$.

30. L.R.F. Rose, Journal of the American Ceramic Society 34 (1986) 208-211.

31. J.C. Amazigo and B. Budiansky, International Journal of Solids and Structures 24 (1988) 751-755.

32. D.M. Stump and B. Budiansky, International Journal of Solids and Structures 25 (1989) 635-646.

33. C.L. Hom and R.M. McMeeking, International Journal of Solids and Structures 26 (1990) 1211-1223.

34. J.C. Lambropoulos, International Journal of Solids and Structures 22 (1986) 1083-1 106.

35. A.E. Giannakopoulos and M. Olsson, Journal of the American Ceramic Society 75 (1992) 2761-2764.

36. J.W. Rudnicki and J.R. Rice, Journal of the Mechanics and Physics of Solids 23 (1975) 371-394.

37. A. Needleman and J.R. Rice, Mechanics of Sheet Metal Forming, D. P. Koistinen and N.-M. Wang (eds.), Plenum Publishing Corporation, New York (1978) 237-267.

38. F.Z. Li and J. Pan, Journal of Applied Mechanics 57 (1990) 40-49.

39. P.E. Reyes-Morel and I.-W. Chen, Journal of the American Ceramic Society 71 (1988) 343-353.

40. P. Dong and J. Pan, International Journal of Solids and Structures 28 (1991) 1113-1127.

41. M.F. Kanninen and C.H. Popelar, Advanced Fracture Mechanics, Oxford University Press, New York (1985).

42. M.L. Williams, Journal of Applied Mechanics 24 (1957) 109-114.

43. G.R. Irwin, Journal of Applied Mechanics 24 (1957) 361-364.

44. P.S. Leevers and J.C. Radon, International Journal of Fracture 19 (1982) 311-325.

45. R.M. McMeeking, Journal of the Mechanics and Physics of Solids 25 (1977) 357-381.

46. M. Kim and J. Pan, Effects of Non-Singular Stresses on Crack-tip Fields for Transformation Toughened Materials: Part 1 Plane Strain Case, presented at the 95th Annual Meeting of the American Ceramic Society, Cincinnati, Ohio (1993). 